\title{
BMJ Open Relationship between serum uric acid, all-cause mortality and cardiovascular mortality in peritoneal dialysis patients: systematic review and meta-analysis of cohort studies
}

\author{
Xue Xue (1) , ${ }^{1,2}$ Chun-Li Lu, ${ }^{2}$ Xin-Yan Jin, ${ }^{2}$ Xue-Han Liu, ${ }^{2}$ Min Yang, ${ }^{3}$ \\ Xiao-Qin Wang, ${ }^{4}$ Hong Cheng, ${ }^{4}$ Jun Yuan, ${ }^{4}$ Qiang Liu, ${ }^{5}$ Ruo-Xiang Zheng, ${ }^{2}$ \\ Nicola Robinson, ${ }^{6}$ Jian-Ping $\mathrm{Liu}^{2}$
}

To cite: Xue X, Lu C-L, Jin X-Y, et al. Relationship between serum uric acid, all-cause mortality and cardiovascular mortality in peritoneal dialysis patients: systematic review and meta-analysis of cohort studies. BMJ Open 2021;11:e052274. doi:10.1136/ bmjopen-2021-052274

- Prepublication history and additional supplemental material for this paper are available online. To view these files, please visit the journal online (http://dx.doi.org/10.1136/ bmjopen-2021-052274).

Received 13 April 2021 Accepted 27 September 2021

Check for updates

(c) Author(s) (or their employer(s)) 2021. Re-use permitted under CC BY-NC. No commercial re-use. See rights and permissions. Published by BMJ.

For numbered affiliations see end of article.

Correspondence to

Dr Jian-Ping Liu;

Liujp@bucm.edu.cn

\section{ABSTRACT}

Objectives To analyse the relationship between serum uric acid (SUA), all-cause and cardiovascular (CV) mortality in peritoneal dialysis (PD) patients to inform clinical practice and future research.

Design A systematic review of observational studies. Data sources PubMed, Embase, Web of Science, the Cochrane Library, China National Knowledge Infrastructure (CNKI), SinoMed, Chinese Science and Technology Journal Database (VIP) and Wan Fang databases were searched from their inception to January 2021 for cohort and casecontrol studies reporting SUA and mortality in patients with PD.

Methods The Newcastle-Ottawa Quality Assessment Scale was used to appraise quality of cohort and case-control studies. Effect estimates were presented as HRs with $95 \%$ Cls in a meta-analysis using STATA V.16.0. Data not suitable for pooling were synthesised qualitatively.

Results Fourteen cohort studies with 24022 patients were included. No case-control studies were identified. For prospective cohort studies, pooled results for the highest SUA category were significantly greater than the lowest for all-cause (one study; 1278participants; HR 1.79; 95\% Cl 1.17 to 2.75) and CV mortality (one study; 1278 participants; HR 2.63; 1.62-4.27). An increase of $1 \mathrm{mg} /$ $\mathrm{dL}$ in SUA level was associated with a $16 \%$ increased risk of all-cause mortality (one study; 1278 participants; HR $1.16 ; 1.03-1.32$ ) and $34 \%$ increased CV mortality risk (one study; 1278 participants; HR 1.34; 1.16-1.55). For retrospective cohort studies, the highest SUA category did not demonstrate an elevated all-cause (five studies; 4570 participants; HR 1.09; 0.70-1.70) or CV mortality (three studies; 3748 participants; HR 1.00; 0.44-2.31) compared with the lowest SUA category. Additionally, there was no increase in all-cause (eight studies; 11541 participants; HR 0.94; 0.88-1.02) or CV mortality (three studies; 7427 participants; HR 0.90; 0.76-1.06) for every $1 \mathrm{mg} / \mathrm{dL}$ increase in SUA level.

Conclusions Results of prospective and retrospective cohort studies were inconsistent. Consequently, prospective, multicentre, long-term follow-up studies are

\section{STRENGTHS AND LIMITATIONS OF THIS STUDY}

$\Rightarrow$ This is the first systematic review of observational studies, which has explored the relationship between serum uric acid level and mortality in patients with peritoneal dialysis.

$\Rightarrow$ We used critical appraisal of included studies and subgroup analysis to present the results and proposed future research directions based on the results.

$\Rightarrow$ Of the included studies, 11 were conducted in China, 2 in other Asian countries and 1 in Europe, this limits the generalisability of our results.

$\Rightarrow$ Dose-response relationship could not be determined from these data.

required to confirm the relationship between SUA and mortality in patients with PD.

\section{INTRODUCTION}

End-stage renal disease (ESRD) is one of the major diseases affecting human health and causes enormous pressure and burden on medical care and society. One of the effective treatments for ESRD is peritoneal dialysis (PD), which is characterised by enabling stable haemodynamics, protecting residual renal function (RRF) and demonstrates good removal of middle molecular toxins and is associated with a low risk of infection and can be delivered at home. ${ }^{1}$ Currently, PD is widely used globally. The total number of people receiving PD worldwide in 2013 reached approximately $220000 .^{2}$ Of concern is that patients with ESRD treated with dialysis still have high mortality. ${ }^{3}$ The identification of potential risk factors has great significance if patients' prognosis and quality of life are to be improved. 
Uric acid (UA) is the final product of purine nucleotide metabolism in humans. Previous studies have demonstrated that elevated serum UA (SUA) is closely related to the increased risk of hypertension, peripheral arterial disease, cardiovascular (CV) event and chronic kidney disease (CKD) in the general population. ${ }^{4-7}$ Higher SUA levels also appear to be an independent risk factor for all-cause and CV mortality in CKD subjects. ${ }^{8}$ However, there have been conflicting results about the relationship between SUA level and risk of death among dialysis patients. In the haemodialysis population, hypouricemia significantly increased mortality. ${ }^{10-12}$ Nevertheless, the role of SUA in all-cause and CV mortality in patients with PD has been controversial. An elevated SUA level has been shown to be an independent risk factor for all-cause and CV mortality in men treated with PD. ${ }^{13}$ Another study showed that the prognostic value of SUA in all-cause and CV mortality was weak in patients with PD. ${ }^{14}$ Hyperuricemia has also been found to predict lower risk of all-cause mortality in patients with PD with lower relative appendicular skeletal muscle. ${ }^{15}$ In short, the effect of SUA on the prognosis of PD patients appears to be inconsistent.

Currently, systematic reviews on the relationship between SUA, all-cause and CV mortality in the PD population are lacking. We hypothesised that there may be an independent correlation between elevated SUA level and mortality in participants with PD. Thus, we systematically analysed available studies to determine whether this hypothesis could be confirmed.

\section{METHODS}

The methods in this review were specified in advance. The review was reported according to the 'Meta-analysis of Observational Studies in Epidemiology guidelines'. ${ }^{16}$

\section{Eligibility criteria}

Types of studies

Cohort and case-control studies were identified.

\section{Participants}

Participants had to receive PD for more than 3 months. There was no restriction on the type of PD, including continuous ambulatory PD, intermittent PD, automated PD, continuous cyclic PD and tidal PD.

\section{Exposure factor}

Hyperuricemia in PD population was the exposure factor in this study. Either categorisation according to baseline SUA level or time-average SUA concentration was acceptable. Definition of hyperuricemia and the categorisation for the SUA level were based on the definition reported in each included article.

\section{Outcome}

The primary outcome was all-cause mortality and death was determined by the hospital medical record or death certificate.
The secondary outcome was CV mortality, defined as a 'CV events': coronary events (myocardial infarction, unstable angina), cardio myopathy, cardiac arrest, cardiac dysrhythmia, congestive heart failure, ischaemic brain injury, cerebrovascular accident and peripheral vascular disease. The cause of death was determined through medical history, hospital medical records or death certificates.

\section{Exclusion criteria}

(1) Unable to obtain the following information from the original article. HR and its corresponding 95\% CIs (or other data required in order perform the calculation) for all-cause or CV mortality for $1 \mathrm{mg} / \mathrm{dL}$ change in SUA level, or for the highest versus lowest SUA category or the lowest versus highest category; (2) cohort studies with a total sample size of less than 100 participants; (3) studies originating from the same cohort sample, or part of a cohort sample that had been previously published. Only the literature that reported the largest sample size and the longest follow-up could be included.

\section{Search strategy}

Two authors (XX and HC) searched the following Chinese and English databases from their inception to 15 January 2021. Chinese databases included China National Knowledge Infrastructure (CNKI), SinoMed, Chinese Science and Technology Journal Database (VIP) and Wan Fang Database. English databases included PubMed, EMBASE, the Cochrane Library and Web of Science. Trial registers including Clinical Trials. gov and the WHO International Clinical Trials Registry Platform were also searched. Additionally, related reviews, conference papers, reference lists and grey literatures were also searched manually. No language or publication type was imposed, published abstracts were also considered. If the retrieved literature had missing information, it was necessary to contact the author via email to obtain the data to ensure that literature could be included. Taking 'PubMed' as an example, the searching strategy was as follows: "Uric Acid" [Mesh] OR "Uric Acid" [Title/Abstract] OR "serum uric acid"[Title/Abstract]) AND ("Mortality"[Mesh] OR "Mortality"[Title/Abstract]) AND ("Peritoneal Dialysis" [Mesh] OR "Peritoneal Dialysis" [Title/Abstract] OR "PD"[Title/Abstract] OR "continuous ambulatory PD" [Title/Abstract] OR "CAPD" [Title/Abstract] OR "intermittent PD"[Title/Abstract] OR "IPD"[Title/Abstract] OR "automated PD"[Title/Abstract] OR "APD"[Title/ Abstract] OR "continuous cyclic PD" [Title/Abstract] OR "CCPD" [Title/Abstract] OR "tidal PD"[Title/Abstract] OR "TPD" [Title/Abstract]). The searching strategies for other databases are presented in online supplemental eTable 1.

\section{Studies selection and data extraction}

The titles and the abstracts were initially screened, then the full-text versions were checked according to the inclusion and exclusion criteria. Two authors (XX and QL) 
examined the full text to identify the eligible studies independently. Two authors (XX and HC) extracted data independently and entered information into a data extraction sheet. Disagreements on study selection and data extraction were resolved by consulting corresponding author J-PL. The following information was extracted from each included study: first author, publication year, age, gender, study design, dialysis duration, sample size, study location, centre, length of follow-up, categories according to SUA, comparison, adjustments and adjusted HR (95\% CI).

\section{Methodological quality assessment}

The Newcastle-Ottawa Quality Assessment Scale (NOS) was used to appraise the quality of observational studies. ${ }^{17}$ NOS allocates a maximum of 9 points for quality of selection, comparability and outcome of study population. Two authors (XX and X-YJ) appraised the quality of included studies independently. Any disagreements were resolved by discussion with corresponding author J-PL. Overall study quality scores were defined as poor $(0-3)$, fair (4-6) or good (7-9).

\section{Statistical analysis}

SUA was analysed not only as a categorical variable but also as a continuous variable in the included studies. The statistical analysis for the overall relationship between SUA level and death risk (all-cause and CV mortality) was based on the random effects model and on comparisons of the highest versus the lowest category of SUA level, or by increase of $1 \mathrm{mg} / \mathrm{dL}$. HR and $95 \%$ CI were used as effect indicators. HR and corresponding 95\% CI of each study were transformed to their natural logarithm (lnHR, $\operatorname{lnlCI}$ and $\ln \mathrm{UCI}$ ), and overall $\mathrm{HR}$ and its $95 \% \mathrm{CI}$ were calculated by exponentiation of the pooled $\operatorname{lnHR}$, lnlCI and $\ln \mathrm{UCI}$.

If data on cases, person-years and HR and 95\% CI for each category were available in the included studies, then a dose-response analysis would be performed to further explore the relationship between SUA and mortality. The potential non-linearity association was examined by modelling SUA levels using restricted cubic splines with three knots at 25, 50 and $75 \%$ of the distribution. We assigned the median or middle point of the upper and lower boundaries in each category as the corresponding dose to the related HR for each study. In general, if there is a dose-response relationship between SUA and mortality, and $p$ value for non-linear $<0.05$, non-linear regression model should be considered. When the $p$ value was close to the critical value of 0.05 , both linear and nonlinear models needed to be fitted.

The $\mathrm{I}^{2}$ was applied to test the statistical heterogeneity among studies (Higgins $e$ t $a l)^{18}$ When $\mathrm{I}^{2}>85 \%$, we believed that the results could not be pooled. Data not suitable for statistical pooling were synthesised qualitatively. To explore the source of heterogeneity among studies, subgroup analyses were conducted according to study design, study location, publication years, adjustment for sex, adjustment for DM and adjustment for albumin. Additionally, the meta-regression analysis was also performed to detect potential heterogeneity based on the above variables when about 10 studies were included. Sensitivity analysis was performed removing one study at a time to explore the robustness of results if data were available. The possibility of publication bias was detected by funnel plots and Egger's test if there were about 10 studies. STATA V.16.0 software (StataCorp, College Station, Texas) was used for data analysis.

\section{Patient and public involvement statement}

Patients or the public WERE NOT involved in the design, or conduct, or reporting or dissemination plans of our study.

\section{RESULTS \\ Search results}

Two hundred and forty-two relevant citations were retrieved. After scanning the full texts, five articles were excluded. Cohort samples from the same study were excluded, ${ }^{131519}$ and only the studies with the largest sample size and the longest follow-up time were included. ${ }^{20}{ }^{21} \mathrm{In}$ addition, a published abstract was excluded, because the total sample size of the entire cohort was only 60 participants. ${ }^{22}$ Another study was excluded due to its pooled effect estimation, which was reported as an OR with $95 \%$ CI. ${ }^{23}$ Finally, 14 studies were eligible for this review. Details of the search and selection process are illustrated in figure 1.

\section{Characteristic of included trials}

A total of 14 studies consisting of 24022 participants were included. $^{14202124-34}$ All were cohort studies, including prospective and retrospective studies. The main characteristics of included studies are given in tables 1 and 2.

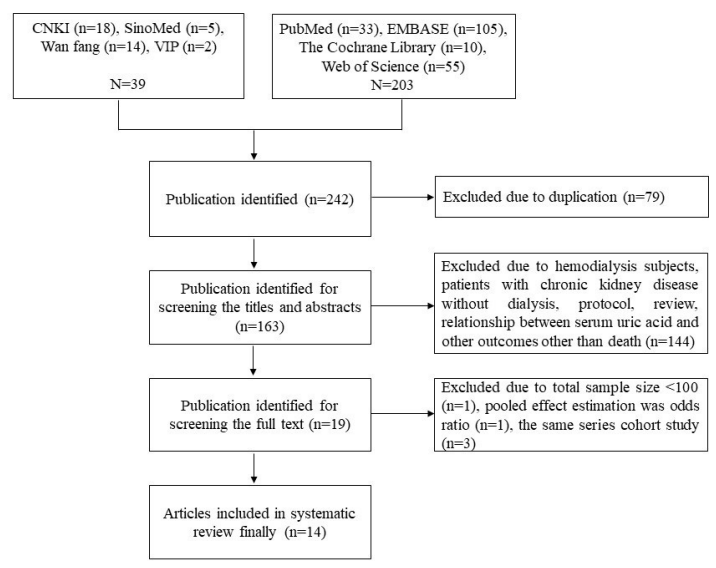

Figure 1 Flow diagram of study search and selection. CNKI, China National Knowledge Infrastructure. VIP, Chinese Science and Technology Journal Database. 


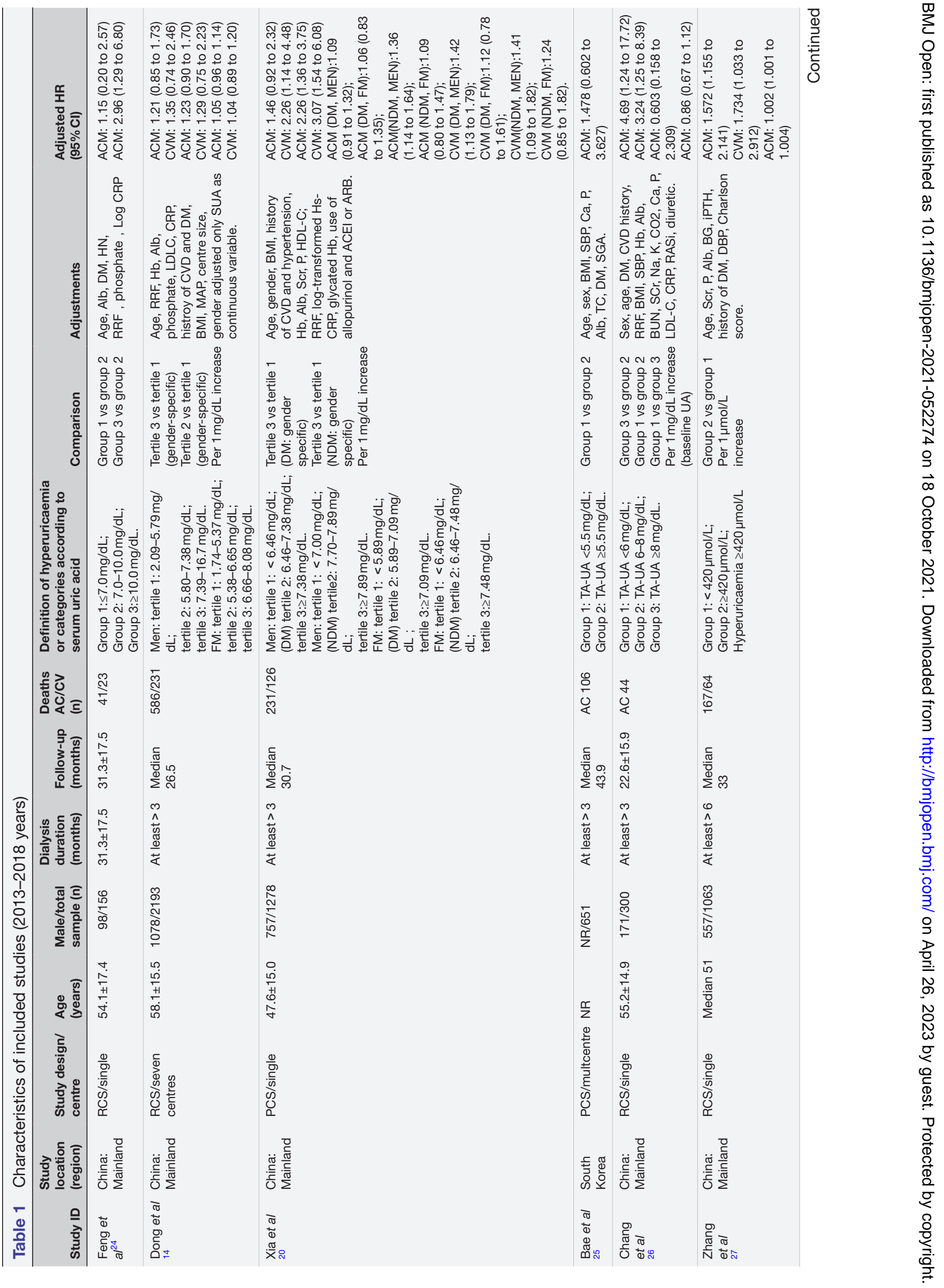




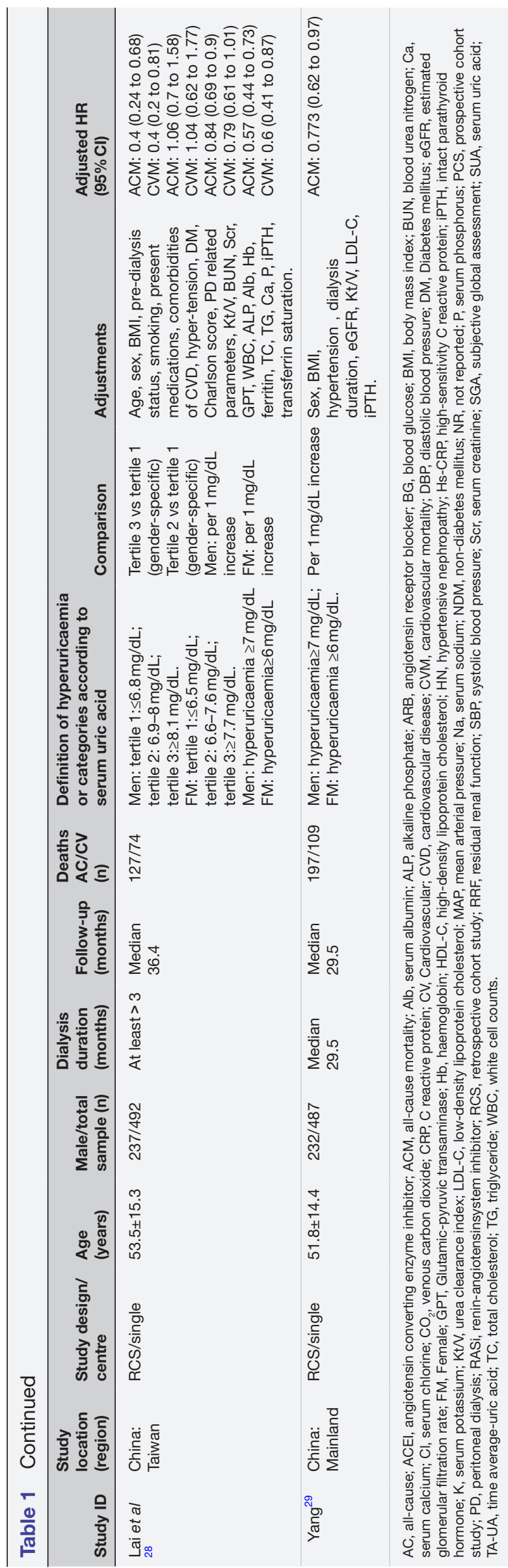

Methodological quality of included studies

The overall quality of included studies was good with a mean NOS score of 7.57 (range 7-9). All studies scored greater than or equal to 7 (online supplemental eTable 2). In terms of 'comparability', the most important confounding factors that required adjustment were estimated glomerular filtration rate or serum creatinine or urinary volume or RRF. The above indicators can reflect the patient's current residual renal function status. In addition, according to the literature and clinical observations, other confounding factors needing adjustment should include gender, age, diabetes history, CV disease (CVD) history, Kt/v (urea clearance index, representing dialysis adequacy), use of UA-lowering drugs and serum albumin (representing nutritional status).

\section{Primary outcome}

Relationship between SUA by categories and all-cause mortality In order to reduce the heterogeneity of methodology, we discussed the results according to different study designs. For prospective cohort studies, the summary of HR and 95\% CI of all-cause mortality for the highest SUA category compared with the lowest category came from one study, which included 1278 patients. ${ }^{20}$ As presented in figure 2, the pooled result of the highest SUA category was significantly higher than the lowest for all-cause mortality (HR $1.79 ; 95 \%$ CI 1.17 to 2.75 ).

In retrospective cohort studies, five studies with 4570 patients reported HR and 95\% CI of all-cause mortality for the highest versus the lowest SUA category. ${ }^{1427283233}$ Allcause mortality (HR 1.09; 95\% CI 0.70 to 1.70 ; figure 2) was not significantly elevated compared with the lowest category of patients with PD.

$\mathrm{HR}$ and corresponding $95 \% \mathrm{CI}$ were reported in three retrospective cohort studies for the lowest versus the highest SUA category. ${ }^{25}{ }^{26}{ }^{30}$ Among them, the data from one article ${ }^{26}$ were supplemented by the corresponding author via e-mail. The pooled HR was 1.52 (95\% CI 0.79 to 2.89 ), with heterogeneity of $\mathrm{I}^{2}=32.8 \%$.

\section{Relationship between SUA per $1 \mathrm{mg} / \mathrm{dL}$ increase and all-cause mortality}

Only one prospective study with 1278 patients with PD reported $\mathrm{HR}$ and $95 \% \mathrm{CI}$ of all-cause mortality for every $1 \mathrm{mg} / \mathrm{dL}$ increase in SUA level. ${ }^{20}$ The pooled result showed that for every $1 \mathrm{mg} / \mathrm{dL}$ increase in SUA level, risk of all-cause death was increased by $16 \%$ (HR 1.16; $95 \%$ CI 1.03 to 1.32 ; figure 3 ).

For the retrospective cohort studies, eight studies with 11541 patients with PD reported HR and 95\% CI of all-cause mortality for every $1 \mathrm{mg} / \mathrm{dL}$ increase in SUA level. ${ }^{142126-293234}$ When the units of SUA concentration in the literature were different, $60 \mu \mathrm{mol} / \mathrm{L}$ was approximately equal to $1 \mathrm{mg} / \mathrm{dL}$. In short, each $1 \mathrm{mg} / \mathrm{dL}$ increase in SUA level did not appear to significantly increase the risk of all-cause mortality in the PD population (HR 0.94; $95 \%$ CI 0.88 to 1.02 ; figure 3 ). 


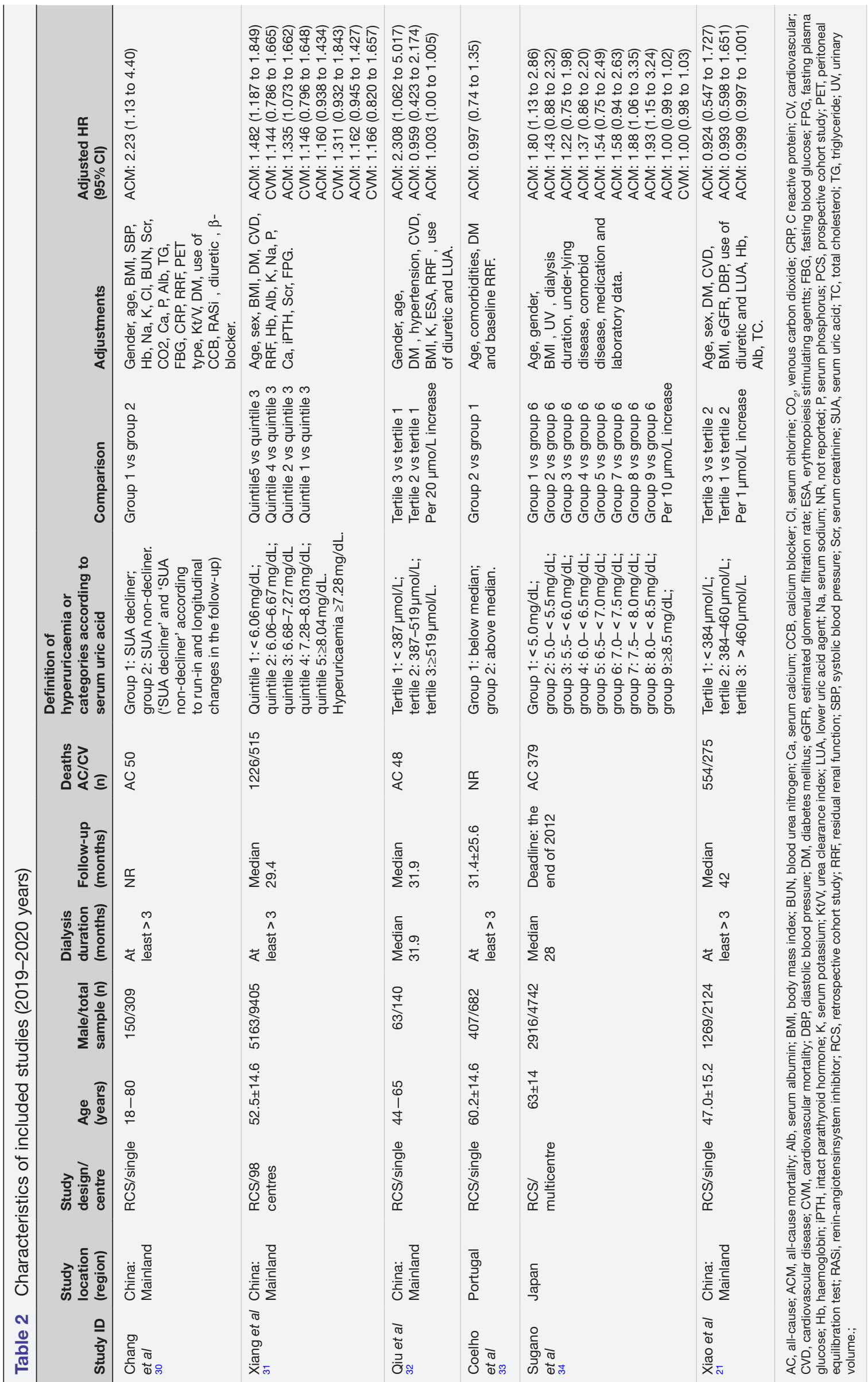




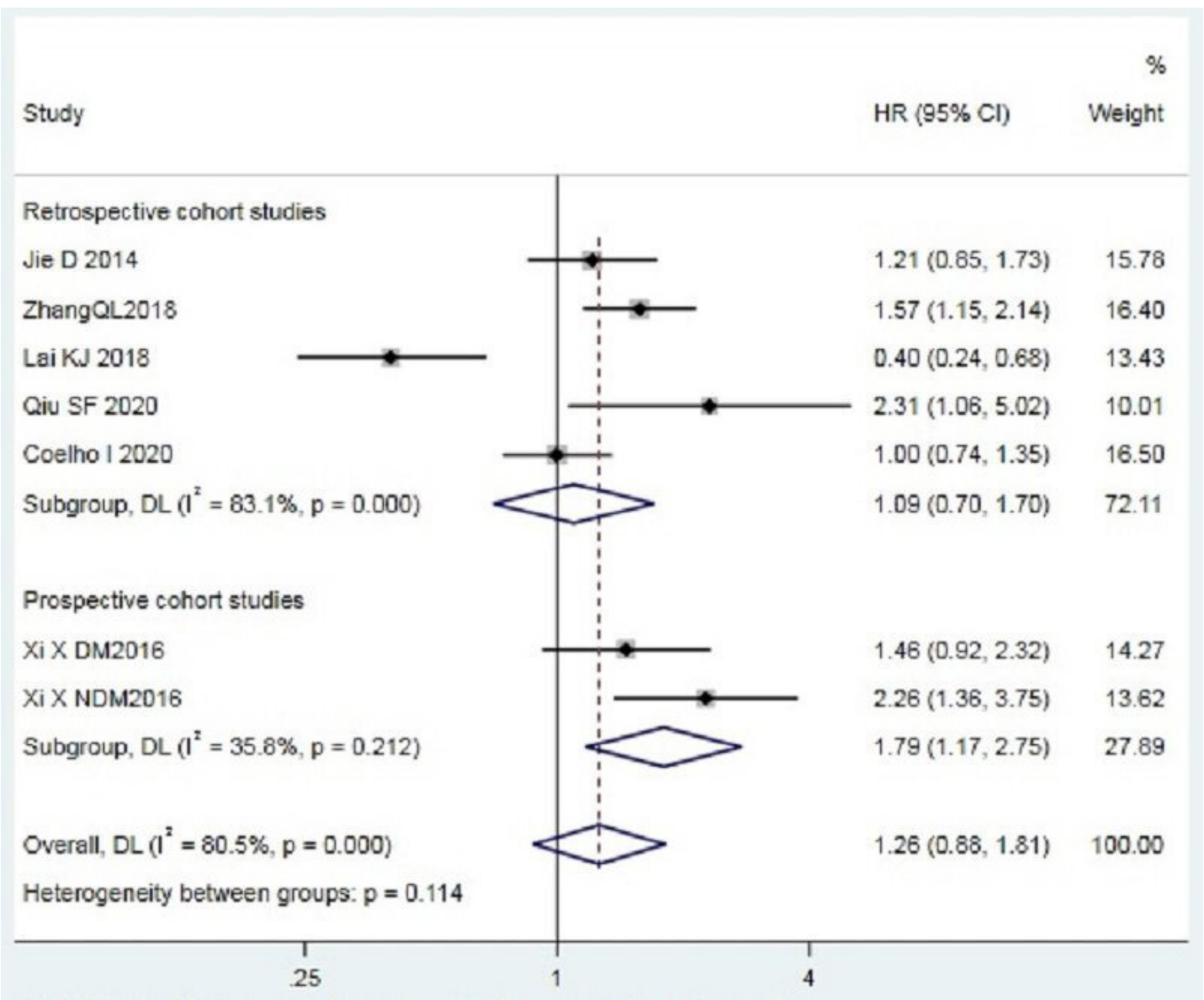

Figure 2 Forest plot and pooled HR for relationship between SUA by categories (the highest SUA category vs the lowest) and all-cause mortality in PD patients. Weights and between-subgroup heterogeneity test are from random-effects model. PD, Peritoneal dialysis; SUA, Serum uric acid.

Dose-response relationship between SUA and all-cause mortality Most of the included studies 14202125272930 32-34 reported only the number of outcomes for the entire cohort population. It was not possible to obtain the number of all-cause and CV deaths and person-years for each category. We tried our best to contact authors by email or phone in order to acquire the necessary data for the non-linearity test, only one author responded and provided relevant data. ${ }^{26} \mathrm{~A}$ dose-response analysis was not possible.

\section{Secondary outcome}

Relationship between SUA by categories and CV mortality

One prospective cohort study with 1278 patients reported HR and $95 \%$ CI of CV mortality for the highest SUA category compared with the lowest. ${ }^{20}$ The pooled result of HR comparing the highest versus the lowest category was 2.63 (95\% CI 1.62 to 4.27 ) (figure 4 ).

Three retrospective cohort studies with 3748 patients reported $\mathrm{HR}$ and $95 \% \mathrm{CI}$ of CV mortality for the highest versus the lowest SUA category. ${ }^{142728}$ The highest SUA category was no more in terms of elevated CV mortality (HR 1.00; 95\% CI 0.44 to 2.31) compared with the lowest category of patients with PD (figure 4).
Relationship between SUA per $1 \mathrm{mg} / \mathrm{dL}$ increase and CV mortality

One prospective cohort study with 1278 patients reported HR and $95 \%$ CI of CV mortality per $1 \mathrm{mg} / \mathrm{dL}$ increase in SUA level. ${ }^{20}$ An increase in each $1 \mathrm{mg} / \mathrm{dL}$ in SUA level was associated with a $34 \%$ increased risk of CV mortality (HR 1.34; $95 \%$ CI 1.16 to 1.55 ) (figure 5 ).

Three retrospective cohort studies with 7427 patients reported HR and $95 \%$ CI of CV mortality per $1 \mathrm{mg} / \mathrm{dL}$ increase in SUA level. ${ }^{142834}$ Meta-analysis showed that each $1 \mathrm{mg} / \mathrm{dL}$ increase in SUA level did not appear to significantly increase the risk of $\mathrm{CV}$ death in $\mathrm{PD}$ population (HR 0.90; 95\% CI 0.76 to 1.06) (figure 5).

\section{Additional analysis}

Subgroup analysis and meta-regression

We explored the source of heterogeneity through subgroup analysis and meta-regression. Subgroup analysis only included literature, which compared the highest versus the lowest category of SUA level or explored a change of $1 \mathrm{mg} / \mathrm{dL}$ increase. The pooled HR (95\% CI) and $\mathrm{I}^{2}$ of subgroup analysis of the relationship between SUA and all-cause and CV mortality are presented in tables 3 and 4 , respectively. 


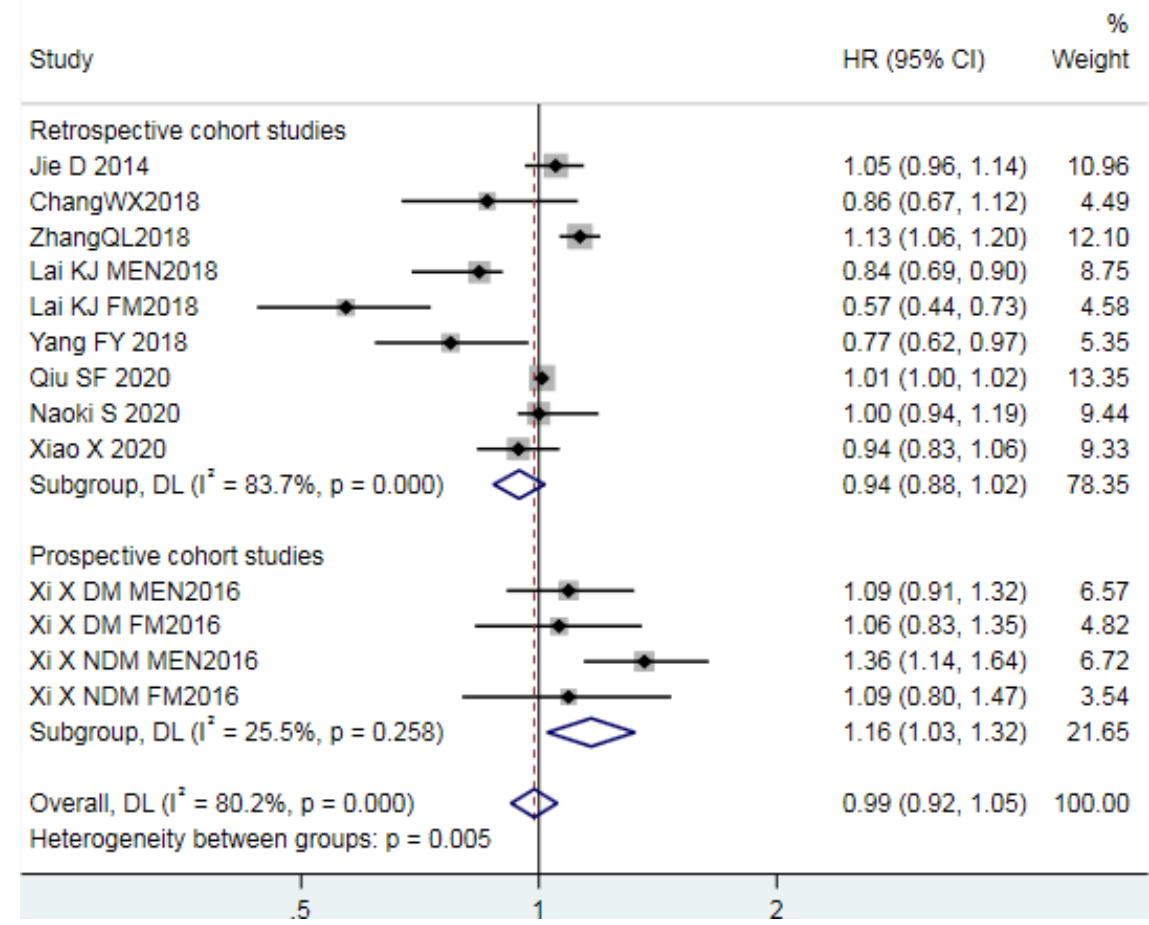

Figure 3 Forest plot and pooled HR for relationship between SUA per $1 \mathrm{mg} / \mathrm{dL}$ increase and all-cause mortality in PD patients. Weights and between-subgroup heterogeneity test from ramdom-effets model. DM, diabetes mellitus; FM, famale; NDM, nondiabetes mellitus; PD, peritoneal dialysis; SUA, serum uric acid.

As mentioned before, whether SUA was a categorical variable or a continuous variable, the results of the prospective cohort study differed from those of retrospective studies. Besides, combined with the results of subgroup analysis, when SUA was estimated as a categorical variable, the association of higher SUA level with increased all-cause and CV mortality was significant in studies from mainland China, but not in other countries. SUA as a continuous variable showed that the relationship of higher CV mortality for $1 \mathrm{mg} / \mathrm{dL}$ increase in

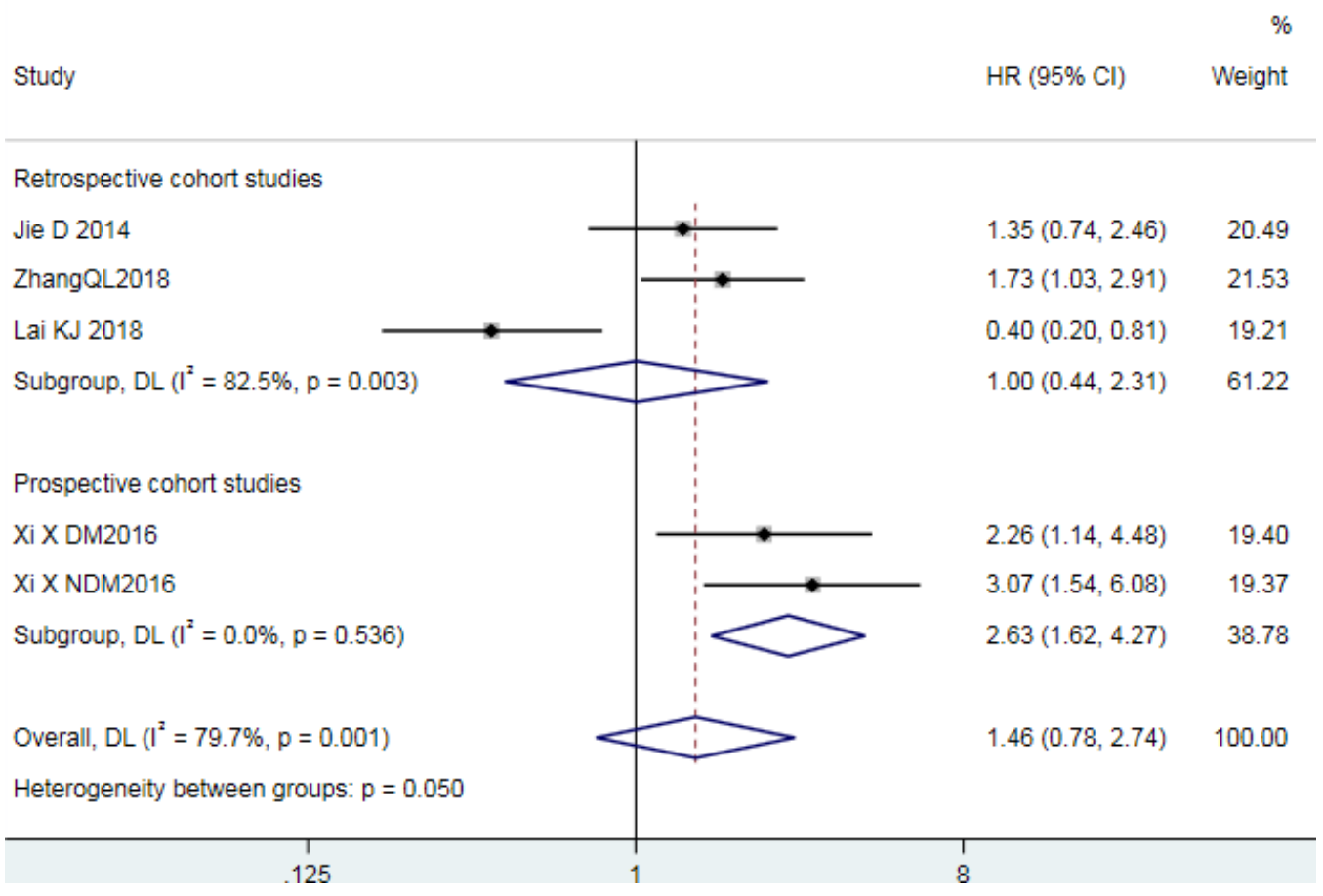

Figure 4 Forest plot and pooled HR for relationship between SUA by categories (the highest SUA category vs the lowest) and cardiovascular mortality in PD patients. Weights and between-subgroup hetetrogeneity test and from random-effects model. PD, peritoneal dialysis; SUA, serum uric acid. 


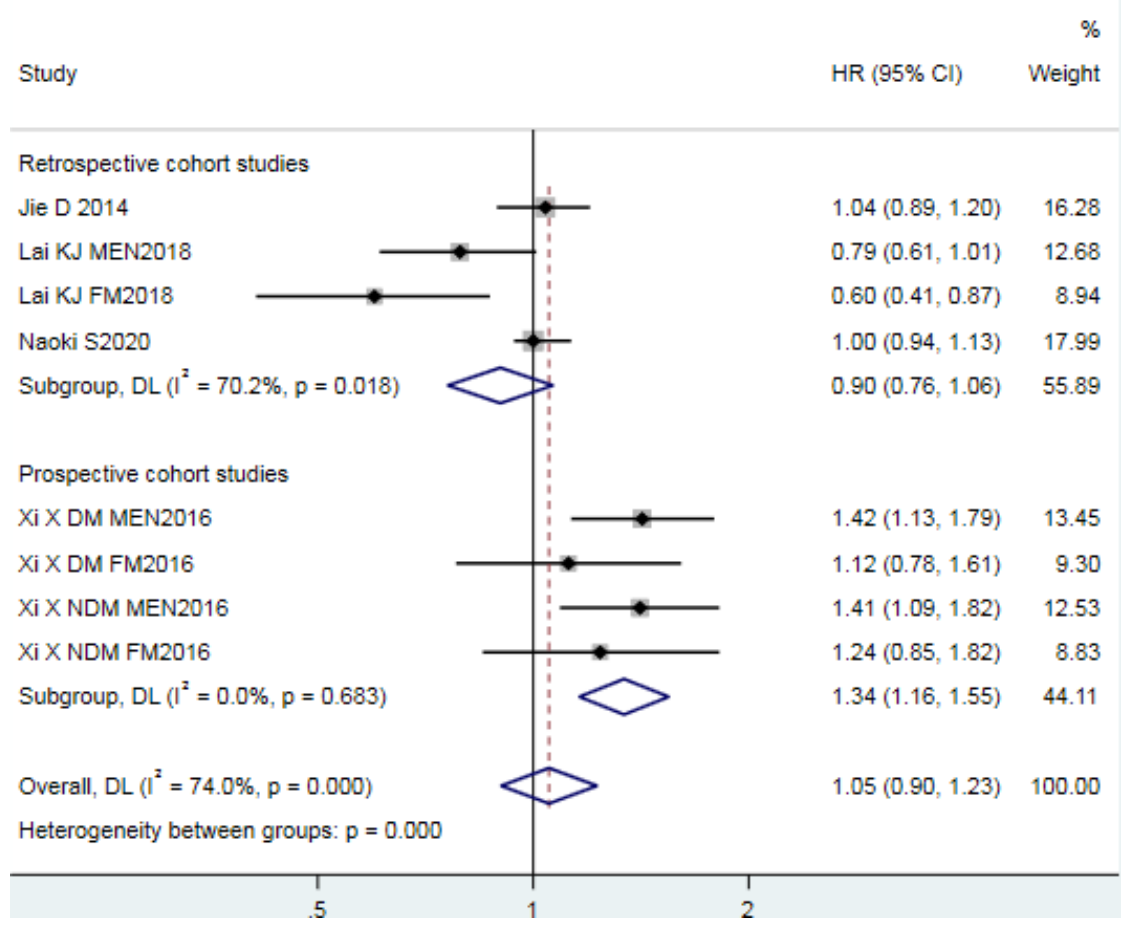

Figure 5 Forest plot and pooled HR for relationship between SUA per $1 \mathrm{mg} / \mathrm{dL}$ increase and cardiovascular mortality in PD patients. Weights and between-subgroup heterogenelty test are from random-effects model. DM, diabetes mellitus; FM, famale; NDM, non-diabetes mellitus; PD, peritoneal dialysis; SUA, serum uric acid.

SUA level was significant in studies from mainland China, but not elsewhere. Furthermore, we analysed the relevant studies published in the past 10 years, and results of studies completed during 2011-2016 were different from the results during 2017-2021 period.

In addition, in studies of the relationship between SUA (as a continuous variable) and all-cause mortality, study design, study location, publication years, adjusted for sex and DM were heterogeneous by meta-regression (table 3).

\section{Test of publication bias}

Funnel plots and Egger's test $(\mathrm{t}=1.07, p=0.309)$ indicated that there was no obvious publication bias of studies for the relationship between all-cause mortality and SUA level per $1 \mathrm{mg} / \mathrm{dL}$ increase. The funnel plot is presented in online supplemental eFigure 1.

\section{Sensitivity analysis}

In retrospective cohort studies, results of primary outcome showed there was no significant effect on the pooled HR values of other studies with one study removed at a time. The above indicated that the results were robust.

\section{DISCUSSION}

\section{Principal findings and comparison with prior reviews}

For PD population, previous original studies indicated inconsistent relationship between SUA and mortality. After searching systematically, we found that there were some meta-analyses investigating the correlation between SUA and mortality in different populations, ${ }^{35-38}$ however, we have not yet found a review only of patients withPD. A systematic review published in 2016 showed that elevated SUA level was significantly associated with the risk of death in patients with CKD, including dialysis and non-dialysis subjects. ${ }^{39}$ Subgroup analysis in this review demonstrated that hyperuricaemia was an independent predictor for mortality in PD population, while, this predictive value was not found in the haemodialysis (HD) population. As only one prospective cohort study and two retrospective cohort studies were included in the subgroup analysis, results should be interpreted with caution.

In our study, we included a total of 14 cohort studies, of which 2 were prospective studies and 12 were retrospective studies. There was no obvious publication bias of studies according to funnel plots and Egger's test. Researchers can not control the process of data accumulation in retrospective cohort studies, but researchers can directly acquire relevant data on exposure and outcome according to the study design in prospective studies, so the risk of bias is small. Thus, instead of pooling results of the two studies, we reported them individually. Only one prospective cohort study suggested that regardless of whether SUA was estimated as a continuous or a categorical variable, elevated SUA level was significantly associated with increased risk of all-cause and CV mortality in patients with $\mathrm{PD}$, whereas, there was no significant association between them in the retrospective studies. Below, we have attempted to discuss the inconsistency of the results from the aspects of participants, exposure, comparability and outcomes. 
Table 3 Subgroup analyses of the relationship between serum uric acid and all-cause mortality

\begin{tabular}{|c|c|c|c|c|c|c|c|c|c|}
\hline & \multicolumn{4}{|c|}{ Serum uric acid (categorical variable) } & \multicolumn{5}{|c|}{ Serum uric acid (continuous variable) } \\
\hline & $\begin{array}{l}\text { Number of } \\
\text { study }\end{array}$ & $\begin{array}{l}\text { Sample } \\
\text { size }\end{array}$ & HR $(95 \% \mathrm{Cl})$ & $\begin{array}{l}\text { Heterogeneity } \\
\left(I^{2}\right)\end{array}$ & $\begin{array}{l}\text { Number of } \\
\text { study }\end{array}$ & $\begin{array}{l}\text { Sample } \\
\text { size }\end{array}$ & HR $(95 \% \mathrm{Cl})$ & $\begin{array}{l}\text { Heterogeneity } \\
\left(I^{2}\right)\end{array}$ & $\begin{array}{l}\text { Meta- } \\
\text { regression } \\
\text { ( } P \text { value) }\end{array}$ \\
\hline Study design & & & & & & & & & $P=0.007$ \\
\hline $\begin{array}{l}\text { Prospective cohort } \\
\text { study }\end{array}$ & 1 & 1278 & 1.79 (1.17 to 2.75$)$ & $35.8 \%$ & 1 & 1278 & 1.16 (1.03 to 1.32$)$ & $25.5 \%$ & \\
\hline $\begin{array}{l}\text { Retrospective cohort } \\
\text { study }\end{array}$ & 5 & 4570 & 1.09 (0.70 to 1.70$)$ & $83.1 \%$ & 8 & 11541 & 0.94 (0.88 to 1.02$)$ & $83.7 \%$ & \\
\hline \multicolumn{10}{|l|}{ Study location } \\
\hline China-mainland & 4 & 4674 & 1.57 (1.26 to 1.96$)$ & $21.1 \%$ & 7 & 7585 & 1.04 (0.97 to 1.11$)$ & $73.0 \%$ & $P<0.001$ \\
\hline China-Taiwan & 1 & 492 & 0.40 (0.24 to 0.68$)$ & - & 1 & 492 & 0.70 (0.48 to 1.03$)$ & $85.9 \%$ & \\
\hline Other & 1 (Portugal) & 682 & $1.00(0.74$ to 1.35$)$ & - & 1 (Japan) & 4742 & 1.00 (0.89 to 1.13$)$ & - & $P=0.002$ \\
\hline \multicolumn{9}{|l|}{ Publication years } & $P=0.017$ \\
\hline $2011-2016$ & 2 & 3471 & 1.53 (1.08 to 2.18$)$ & $48.9 \%$ & 2 & 3471 & $1.12(1.01$ to 1.24$)$ & $37.9 \%$ & \\
\hline $2017-2021$ & 4 & 2377 & 1.07 (0.59 to 1.93$)$ & $87.3 \%$ & 7 & 9348 & 0.92 (0.84 to 1.01$)$ & $85.5 \%$ & \\
\hline \multicolumn{9}{|l|}{ Number of centre } & $P=0.539$ \\
\hline Single centre & 5 & 3655 & 1.27 (0.82 to 1.98$)$ & $83.7 \%$ & 7 & 5884 & 0.97 (0.89 to 1.06$)$ & $83.3 \%$ & \\
\hline Multicentre & 1 & 2193 & 1.21 (0.85 to 1.73$)$ & - & 2 & 6935 & 1.03 (0.96 to 1.11$)$ & $0 \%$ & \\
\hline \multicolumn{9}{|l|}{ Adjusted for sex } & $P<0.001$ \\
\hline Yes & 4 & 4103 & 1.27 (0.72 to 2.26$)$ & $84.9 \%$ & 8 & 11756 & 0.97 (0.90 to 1.04$)$ & $76.7 \%$ & \\
\hline No & 2 & 1745 & 1.25 (0.80 to 1.95$)$ & $76.7 \%$ & 1 & 1063 & $1.13(1.06$ to 1.20$)$ & - & \\
\hline \multicolumn{9}{|c|}{ Adjusted for diabetes mellitus } & $P=0.019$ \\
\hline Yes & 6 & 5848 & 1.26 (0.88 to 1.81$)$ & $80.5 \%$ & 8 & 12332 & 1.00 (0.94 to 1.07$)$ & $80.0 \%$ & \\
\hline No & 0 & 0 & - & - & 1 & 487 & 0.77 (0.62 to 0.97$)$ & - & \\
\hline \multicolumn{9}{|c|}{ Adjusted for serum albumin } & $P=0.108$ \\
\hline Yes & 4 & 5026 & 1.22 (0.76 to 1.96$)$ & $84.7 \%$ & 7 & 12192 & 0.99 (0.90 to 1.09$)$ & $81.0 \%$ & \\
\hline No & 2 & 822 & 1.40 (0.62 to 3.14$)$ & $74.4 \%$ & 2 & 627 & 0.90 (0.70 to 1.17$)$ & $81.6 \%$ & \\
\hline
\end{tabular}

First of all, the prospective cohort study clearly indicated that participants on PD were consecutively enrolled. It is well known that consecutive recruitment is very important to reduce selection bias. While, in some retrospective studies, the process of enrolment was not detailed. The follow-up of the participants was also a prominent issue, including the duration and adequacy of follow-up and the rate of loss to follow-up. In a prospective study, effective measures can be taken to reduce the loss to follow-up rate to avoid bias. The rate of loss to follow-up in a prospective study by Xia X et al was only $3.5 \%,{ }^{20}$ but in some retrospective cohorts, the adequacy and lost follow-up rates were not reported.

Second, hyperuricaemia in the PD population was the exposure factor of this study. Both prospective and retrospective cohort studies, the definition of hyperuricaemia and the categorisation for the SUA level were based on the definition provided in each included article. It should be noted that in retrospective multicentre studies, the measurement methods of SUA may not be uniform across centres. This may lead to measurement bias and have a slight impact on results.

Third, control of the most important and other confounding factors is very important for the comparability between the groups. The most important confounding factors included indicators that can reflect the patient's current residual renal function status. Other confounding factors need to be adjusted and should include gender, age, diabetes history, CVD history, Kt/V, use of UA-lowering drugs and serum albumin. Most of the studies did not adjust for all potential risk factors. For example, the prospective study of Xia X et al lacked adjustment for the confounding factor $\mathrm{Kt} / \mathrm{V} .^{20}$ Therefore, we can not exclude the potential impact of these uncontrolled confounding factors.

Regarding the outcomes, the definition of all-cause and CV death was clear. However, the handling of patients transferring to haemodialysis therapy, loss to follow-up and renal transplantation was different for prospective and retrospective studies. The above information from patients was used as censoring data for survival analysis in the prospective study, ${ }^{20}$ whereas, in some retrospective studies, they were directly excluded from the study. ${ }^{32}$ This may affect the results and lead to inconsistencies between the prospective and retrospective studies. Although the risk of bias in the design type of prospective study was relatively small, the interpretation of the results should still be cautious due to the limited quality and quantity of prospective study.

SUA, known for its detrimental effect, is an endothelial toxin and plays a role in endothelial dysfunction. ${ }^{40}$ However, as a powerful free radical scavenger in human 
Table 4 Subgroup analyses of the relationship between serum uric acid and cardiovascular mortality

\begin{tabular}{|c|c|c|c|c|c|c|c|c|}
\hline & \multicolumn{4}{|c|}{ Serum uric acid (categorical variable) } & \multicolumn{4}{|c|}{ Serum uric acid (continuous variable) } \\
\hline & $\begin{array}{l}\text { Number } \\
\text { of study }\end{array}$ & $\begin{array}{l}\text { Sample } \\
\text { size }\end{array}$ & HR $(95 \% \mathrm{Cl})$ & $\begin{array}{l}\text { Heterogeneity } \\
\left(I^{2}\right)\end{array}$ & $\begin{array}{l}\text { Number } \\
\text { of study }\end{array}$ & $\begin{array}{l}\text { Sample } \\
\text { size }\end{array}$ & HR (95\% Cl) & $\begin{array}{l}\text { Heterogeneity } \\
\left(I^{2}\right)\end{array}$ \\
\hline \multicolumn{9}{|l|}{ Study design } \\
\hline Prospective cohort study & 1 & 1278 & 2.63 (1.62 to 4.27$)$ & $0.0 \%$ & 1 & 1278 & 1.34 (1.16 to 1.55$)$ & $0.0 \%$ \\
\hline Retrospective cohort study & 3 & 3748 & $1.00(0.44$ to 2.31$)$ & $82.5 \%$ & 3 & 7427 & 0.90 (0.76 to 1.06$)$ & $70.2 \%$ \\
\hline \multicolumn{9}{|l|}{ Study location } \\
\hline China-Taiwan & 1 & 492 & $0.40(0.20$ to 0.80$)$ & - & 1 & 492 & $0.71(0.55$ to 0.93$)$ & $29.5 \%$ \\
\hline Other & & & & & 1 (Japan) & 4742 & $1.00(0.91$ to 1.10$)$ & - \\
\hline \multicolumn{9}{|l|}{ Publication years } \\
\hline $2011-2016$ & 2 & 3471 & 2.06 (1.27 to 3.34$)$ & $38.7 \%$ & 2 & 3471 & 1.22 (1.05 to 1.43$)$ & $44.6 \%$ \\
\hline $2017-2021$ & 2 & 1555 & 0.85 (0.20 to 3.57$)$ & $90.8 \%$ & 2 & 5234 & $0.82(0.62$ to 1.08$)$ & $77.7 \%$ \\
\hline \multicolumn{9}{|l|}{ Adjusted for sex } \\
\hline Yes & 3 & 3963 & 1.39 (0.60 to 3.24$)$ & $84.4 \%$ & 4 & 8705 & 1.05 (0.90 to 1.23$)$ & $74.0 \%$ \\
\hline No & 1 & 1063 & 1.73 (1.03 to 2.91$)$ & - & 0 & 0 & - & - \\
\hline \multicolumn{9}{|l|}{ Adjusted for diabetes mellitus } \\
\hline Yes & 4 & 5026 & $1.46(0.78$ to 2.74$)$ & $79.7 \%$ & 4 & 8705 & 1.05 (0.90 to 1.23$)$ & $74.0 \%$ \\
\hline No & 0 & 0 & - & - & 0 & 0 & - & - \\
\hline \multicolumn{9}{|l|}{ Adjusted for serum albumin } \\
\hline Yes & 4 & 5026 & 1.46 (0.78 to 2.74$)$ & $79.7 \%$ & 4 & 8705 & 1.05 (0.90 to 1.23$)$ & $74.0 \%$ \\
\hline No & 0 & 0 & - & - & 0 & 0 & - & - \\
\hline
\end{tabular}

at the same time, SUA may be expected to offer a number of benefits within the CV system in patients with PD. ${ }^{41} 42$ Besides, the problem of protein loss and malnutrition is prominent in PD population. ${ }^{43}$ 'Malnutritioninflammation complex syndrome (MICS)' is believed to be the main cause of the high rate of CV atherosclerotic disease and increased mortality and hospitalisation in patients with $\mathrm{HD}^{44}{ }^{45}$ The underlying mechanism of MICS may also be present in patients with PD. As a nutritional marker, SUA might be involved in the MICS axis. Therefore, the relationship between SUA and mortality in patients with $\mathrm{PD}$ is a complex proposition. Taking into account the feature of SUA itself, we hypothesise that both extremely low and high SUA level may increase the risk of death. In our study, we also would like to explore the dose-response relationship between SUA level and mortality in PD population, but in the end the analysis to explore a dose-response relationship was impossible due to insufficient data. Further investigations are warranted to clarify this relationship and explore the range of SUA concentration associated with the lowest mortality in patients with PD.

Moreover, in addition to different study designs, different study location was also one of the main sources of heterogeneity among studies according to the metaregression test. Subgroup analysis results further suggested hyperuricaemia was associated with a high risk of $\mathrm{CV}$ death in PD population only in mainland China. As a result, the relationship between SUA level and the risk of death in different regions needs to be explored and verified by prospective studies in future.

\section{Implications further research}

Since the results of prospective and retrospective cohort studies were inconsistent, and the different regions seemed to lead to different results, prospective, multicentre, long-term follow-up studies are required in future. It is important to explore the relationship between SUA level and the risk of death in different regions as well as to determine the range of SUA concentrations, which can reduce mortality and improve prognosis in patients with PD.

Additionally, since patients with PD often suffer from underlying diseases and complex conditions, adjustment is required for confounding factors to explore the relationship between these factors and prognosis. For the PD population, the following confounding factors should be considered to make the evidence more compelling, such as: traditional independent risk factors of $\mathrm{CV}$ events (age, gender, total lipoprotein cholesterol, low or highdensity lipoprotein cholesterol, hypertension, diabetes, smoking ${ }^{46}$ ), history of $\mathrm{CV}$, emotion status, residual renal function, the related parameters of $\mathrm{PD}$, the parameters 
of nutritional status, use of diuretic and lower UA agents, etc.

Comprehensive information should be reported in detail in the study report, so that readers can become more familiar with the details of the study and can conduct secondary research to avoid wasting research resources.

\section{Study limitations}

There were several limitations in this review. Systematic reviews of observational studies can provide a higher level of evidence, but they also have methodological limitations. For example, the included original studies may differ in their design, data collection methods and definitions of exposure, confounding factors and outcomes. These differences may have a slight influence on the true effect size. Second, in this review, the included studies were mainly from Asian populations (only one from Europe), and the generalisability of the results was limited. Third, in spite of many important confounding factors that to be adjusted in the studies, residual and unknown confounding factors can not be entirely excluded. Fourth, the duration of follow-up in all studies was less than 5 years. It is difficult to determine long-term impact of elevated SUA level on mortality. Finally, some necessary data were not obtained, and the exploration of dose-response relationship could not be conducted but will need to be determined in future studies.

\section{CONCLUSIONS}

The results from the prospective and retrospective cohort studies were inconsistent. Only one prospective cohort study showed that elevated SUA level was significantly associated with increased risk of all-cause and CV mortality in patients with PD. Nevertheless, the pooled result of retrospective cohort studies did not appear to indicate a prominent association. So it is necessary to use SUA-lowering agents with caution for patients with PD in clinics. International prospective, multicentre, long-term follow-up studies are needed in the future to investigate the relationship between SUA level and the risk of death and to explore the range of SUA concentrations associated with the lowest mortality in patients with PD.

\section{Author affiliations}

${ }^{1}$ First Clinical College and Affiliated Hospital, Hubei University of Traditional Chinese Medicine, Wuhan, China

${ }^{2}$ Centre for Evidence-Based Chinese Medicine, Beijing University of Chinese Medicine, Beijing, China

${ }^{3}$ Basic Medical School, Hubei University of Traditional Chinese Medicine, Wuhan, China

${ }^{4}$ Department of Nephrology, Hubei Provincial Hospital of Traditional Chinese Medicine, Wuhan, China

${ }^{5}$ Department of Cardiology, Hubei Provincial Hospital of Traditional Chinese Medicine, Wuhan, China

${ }^{6}$ Institute of Health and Social Care, London South Bank University, London, UK

Acknowledgements Centre for Evidence-Based Chinese Medicine of Beijing University of Chinese Medicine hosted the study and provided learning guidance to domestic visiting scholars. Thanks to the National Administration of Traditional Chinese Medicine for the base construction project. We greatly thanked Fang-fang
Zhao from Xiyuan Hospital, China Academy of Chinese Medical Sciences, for the suggestion during the revision process.

Contributors Research idea and study design: $X X$ and J-PL. Protocol and registration: $X X, C-L L, X-Y J$ and $X-H L$. Acquisition, analysis or interpretation of data: $X X, H C, Q L, J Y$ and J-PL. Drafting of the manuscript: XX, X-HL, C-LL, X-YJ and R-XZ Statistical analysis: MY, XX and X-QW. Critical revision of the manuscript: $X-Q W, N R$ and J-PL. Supervision: J-PL.

Funding This work was supported by a grant from the key project of the National Natural Science Foundation of China (number 81830115), and a grant from the National Natural Science Foundation of China (number 81874439, 82074364). Prof. Nicola Robinson (visiting professor of Beijing University of Chinese Medicine) is funded by International development and capacity enhancement of evidencebased Chinese medicine Project, Ministry of Science and Technology of the People's Republic of China (G20200001187).

Competing interests None declared.

Patient consent for publication Not applicable.

Provenance and peer review Not commissioned; externally peer reviewed.

Data availability statement Data are available upon reasonable request. All data relevant to the study are included in the article or uploaded as supplementary information. The datasets used for meta-analyses are available from the corresponding author on reasonable request.

Supplemental material This content has been supplied by the author(s). It has not been vetted by BMJ Publishing Group Limited (BMJ) and may not have been peer-reviewed. Any opinions or recommendations discussed are solely those of the author(s) and are not endorsed by BMJ. BMJ disclaims all liability and responsibility arising from any reliance placed on the content. Where the content includes any translated material, BMJ does not warrant the accuracy and reliability of the translations (including but not limited to local regulations, clinical guidelines, terminology, drug names and drug dosages), and is not responsible for any error and/or omissions arising from translation and adaptation or otherwise.

Open access This is an open access article distributed in accordance with the Creative Commons Attribution Non Commercial (CC BY-NC 4.0) license, which permits others to distribute, remix, adapt, build upon this work non-commercially, and license their derivative works on different terms, provided the original work is properly cited, appropriate credit is given, any changes made indicated, and the use is non-commercial. See: http://creativecommons.org/licenses/by-nc/4.0/.

ORCID iD

Xue Xue http://orcid.org/0000-0003-1592-7768

\section{REFERENCES}

1 Li PK-T, Chow KM, Van de Luijtgaarden MWM, et al. Changes in the worldwide epidemiology of peritoneal dialysis. Nat Rev Nephrol 2017;13:90.

2 Current status and challenges of peritoneal dialysis treatment. Chinese J Nephrol Dialysis Transplant 2015;24:186-9.

3 Bloembergen WE, Port FK, Mauger EA, et al. A comparison of mortality between patients treated with hemodialysis and peritoneal dialysis. J Am Soc Nephrol 1995;6:177-83.

4 Johnson RJ, Kang D-H, Feig D, et al. Is there a pathogenetic role for uric acid in hypertension and cardiovascular and renal disease? Hypertension 2003;41:1183-90.

5 Shankar A, Klein BEK, Nieto FJ, et al. Association between serum uric acid level and peripheral arterial disease. Atherosclerosis 2008;196:749-55.

6 Gagliardi ACM, Miname MH, Santos RD. Uric acid: a marker of increased cardiovascular risk. Atherosclerosis 2009;202:11-17.

7 Ryoo J-H, Choi J-M, Oh C-M, et al. The association between uric acid and chronic kidney disease in Korean men: a 4-year follow-up study. J Korean Med Sci 2013;28:855.

8 Madero M, Sarnak MJ, Wang X, et al. Uric acid and long-term outcomes in CKD. Am J Kidney Dis 2009;53:796-803.

9 Kanbay M, Yilmaz MI, Sonmez A, et al. Serum uric acid independently predicts cardiovascular events in advanced nephropathy. Am J Nephrol 2012;36:324-31.

$10 \mathrm{Li} \mathrm{M}$, Ye Z-C, Li C-M, et al. Low serum uric acid levels increase the risk of all-cause death and cardiovascular death in hemodialysis patients. Ren Fail 2020;42:315-22.

11 Kim CS, Jin D-C, Yun YC, et al. Relationship between serum uric acid and mortality among hemodialysis patients: retrospective 
analysis of Korean end-stage renal disease registry data. Kidney Res Clin Pract 2017;36:368-76.

12 Latif W, Karaboyas A, Tong L, et al. Uric acid levels and all-cause and cardiovascular mortality in the hemodialysis population. Clin J Am Soc Nephrol 2011;6:2470-7.

13 Xia X, He F, Wu X, et al. Relationship between serum uric acid and all-cause and cardiovascular mortality in patients treated with peritoneal dialysis. Am J Kidney Dis 2014;64:257-64.

14 Dong J, Han Q-F, Zhu T-Y, et al. The associations of uric acid, cardiovascular and all-cause mortality in peritoneal dialysis patients. PLoS One 2014;9:e82342.

15 Xiao X, Yi C, Peng Y, et al. The association between serum uric acid and appendicular skeletal muscle mass and the effect of their interaction on mortality in patients on peritoneal dialysis. Kidney Blood Press Res 2020;45:969-81.

16 Stroup DF, Berlin JA, Morton SC, et al. Meta-analysis of observational studies in epidemiology: a proposal for reporting. meta-analysis of observational studies in epidemiology (moose) group. JAMA 2000;283:2008-12.

17 Wells GA, Shea B, O'Connell D. The Newcastle-Ottawa scale (NOS) for assessing the quality of nonrandomised studies in meta-analyses 2000.

18 Higgins JPT, Thompson SG, Deeks JJ, et al. Measuring inconsistency in meta-analyses. BMJ 2003;327:557-60.

19 Li W, Xiong L, Fan L, et al. Association of baseline, longitudinal serum high-sensitive $\mathrm{C}$-reactive protein and its change with mortality in peritoneal dialysis patients. BMC Nephrol 2017;18:1-10.

20 Xia X, Zhao C, Peng FF, et al. Serum uric acid predicts cardiovascular mortality in male peritoneal dialysis patients with diabetes. Nutr Metab Cardiovasc Dis 2016:26:20-6.

21 Xiao X, Ye H-J, Peng Y, et al. Effect of the interaction between estimated glomerular filtration rate and serum uric acid on all cause and cardiovascular mortality in patients on peritoneal dialysis. Chinese J Nephrol 2020;36:703-10.

22 Stepanova N, Burdeyna O, Snisar L. FP587HYPERURICEMIA is associated with cardiovascular events and all-cause mortality in peritoneal dialysis patients. Nephrol Dial Transplant 2019;34:gfz106. FP587.

23 The effect of serum uric acid level on the prognosis of maintenance peritoneal dialysis patients. J Clin Res 2020;37:1589-91.

24 Feng S, Jiang L, Shi Y, et al. Uric acid levels and all-cause mortality in peritoneal dialysis patients. Kidney Blood Press Res 2013;37:181-9.

25 Bae E, Cho H-J, Shin N, et al. Lower serum uric acid level predicts mortality in dialysis patients. Medicine 2016;95:e3701.

26 Chang W, Zhang W, Wang X, et al. The association of longitudinal serum uric acid and all-cause mortality in incident peritoneal dialysis patients. Blood Purif 2019;47:185-92.

27 Zhang QL, Wang JN, Wang YM. Effects of serum uric acid level on all-cause death and cardiovascular death in patients of maintaining peritoneal dialysis. Chinese J Nephrol 2018;34:809-15.

28 Lai K-J, Kor C-T, Hsieh Y-P. An inverse relationship between hyperuricemia and mortality in patients undergoing continuous ambulatory peritoneal dialysis. J Clin Med 2018;7:416.

29 Yang FY. The associations of time-averaged serum uric acid with cardiovascular mortality and all-cause mortality in peritoneal dialysis patients. Ningxia Medical University, 2018.
30 Chang W, Uchida S, PP Q. Decline in serum uric acid predicts higher risk for mortality in peritoneal dialysis patients - a propensity score analysis. J Nephrol 2019:1-9.

31 Xiang $\mathrm{S}$, Zhang $\mathrm{X}$, Xie $\mathrm{X}$, et al. High serum uric acid level is a mortality risk factor in peritoneal dialysis patients: a retrospective cohort study. Nutr Metab 2019;16:1-10.

32 Qiu SF, Teng J, Shen B. Correlation of serum uric acid before dialysis and all-cause mortality in peritoneal dialysis patients. Chinese $J$ Clin Med 2020;27:815-21.

33 Coelho ID, Rodriguez C, Rodriguez-Carmona A. Serum uric acid, mortality, and decline of residual kidney function in patients undergoing chronic peritoneal dialysis. J Am Soc Nephrol 2020;31:420.

34 Sugano N, Maruyama Y, Ohno I, et al. Effect of uric acid levels on mortality in Japanese peritoneal dialysis patients. Perit Dial Int 2021;41:320-7.

35 Zhao G, Huang L, Song M, et al. Baseline serum uric acid level as a predictor of cardiovascular disease related mortality and all-cause mortality: a meta-analysis of prospective studies. Atherosclerosis 2013;231:61-8.

36 Wang $\mathrm{R}$, Song $\mathrm{Y}$, Yan $\mathrm{Y}$, et al. Elevated serum uric acid and risk of cardiovascular or all-cause mortality in people with suspected or definite coronary artery disease: a meta-analysis. Atherosclerosis 2016;254:193-9.

37 Tamariz L, Harzand A, Palacio A, et al. Uric acid as a predictor of allcause mortality in heart failure: a meta-analysis. Congest Heart Fail 2011;17:25-30

38 Li L, Yang C, Zhao Y, et al. Is hyperuricemia an independent risk factor for new-onset chronic kidney disease?: a systematic review and meta-analysis based on observational cohort studies. BMC Nephrol 2014;15:1-12.

39 Xia X, Luo Q, Li B, et al. Serum uric acid and mortality in chronic kidney disease: a systematic review and meta-analysis. Metabolism 2016:65:1326-41.

40 Yu M-A, Sánchez-Lozada LG, Johnson RJ, et al. Oxidative stress with an activation of the renin-angiotensin system in human vascular endothelial cells as a novel mechanism of uric acidinduced endothelial dysfunction. J Hypertens 2010;28:1234-42.

41 Nieto FJ, Iribarren C, Gross MD, et al. Uric acid and serum antioxidant capacity: a reaction to atherosclerosis? Atherosclerosis 2000;148:131-9.

42 Suzuki T. Nitrosation of uric acid induced by nitric oxide under aerobic conditions. Nitric Oxide 2007;16:266-73.

43 Sánchez-Villanueva R, Bajo A, Del Peso G, et al. Higher daily peritoneal protein clearance when initiating peritoneal dialysis is independently associated with peripheral arterial disease (PAD): a possible new marker of systemic endothelial dysfunction? Nephrol Dial Transplant 2009;24:1009-14.

44 Hur I, Choi SJ, Kalantar-Zadeh K. Serum uric acid and mortality risk among maintenance hemodialysis patients. Kidney Res Clin Pract 2017;36:302-4

45 Kalantar-Zadeh K, Ikizler TA, Block G, et al. Malnutrition-inflammation complex syndrome in dialysis patients: causes and consequences. Am J Kidney Dis 2003;42:864-81.

46 Dessein PH, Joffe BI, Veller MG, et al. Traditional and nontraditional cardiovascular risk factors are associated with atherosclerosis in rheumatoid arthritis. J Rheumatol 2005;32:435-42. 